\title{
Multiple information fusion based on single component feature and diagnosis of gear's early fault
}

\author{
Jianmin Mei ${ }^{1}$, Gang Ren ${ }^{2}$, Chun Chang ${ }^{3}$ \\ Department of Equipment Guarantee, Military Transportation University, Tianjin, 300161, China \\ ${ }^{1}$ Corresponding author \\ E-mail: ${ }^{1}$ meijianmin126@126.com, ${ }^{2}$ gangren@163.com, ${ }^{3}$ chunchang@126.com
}

Received 30 April 2018; accepted 7 May 2018

DOI https://doi.org/10.21595/vp.2018.19936

Check for updates

Copyright (C) 2018 Jianmin Mei, et al. This is an open access article distributed under the Creative Commons Attribution License, which permits unrestricted use, distribution, and reproduction in any medium, provided the original work is properly cited.

\begin{abstract}
A method of multiple information fusion based on single component feature (MIFSCF) is proposed and applied to diagnose early fault of gear. Firstly, meshing frequency component is separated by Fractional Fourier Transform (FRFT) as single component signal, whose order envelop demodulation spectrum (OEDS) is calculated next, and the value of OEDS at feature order of gear's fault is selected as feature parameter. Feature parameters of different sensors are fused by radar graph and analysis results show that MIFSCF is more effective and accurate than the method based on single sensor in gear fault diagnosis.
\end{abstract}

Keywords: FRFT, single components feature, multiple information fusion, fault diagnose of gear.

\section{Introduction}

Fault feature of transmission's early failure is very weak, which can be exposed more evident by variable rotational speed process, but the noise is also stronger, so how to separate target order component from other components and noise is important. Fault feature information included in different signal sampled at different position on the transmission is not the same, it is not accurate if only one position's signal is used to diagnose fault.

Meshing frequency components of rapid acceleration are almost the multiple chirp signal, the time and frequency distribution are so crossed that difficult to be separated in either time domain or frequency domain. Fractional Fourier Transform (FRFT) is especially effective in separating multiple chirp signal, which can extract target chirp component from other components and noise as long as the order is correct in [1-6]. Reference [7, 8] adopted holographic spectrum fusing horizontal and vertical sensor's signal to improve diagnostic reliability, but it can't fuse more than two sensor's signal. Radar graph can fuse many components into one figure to compare the difference of different conditions, so it is suitable for more sensor information fusion.

In order to reduce interference of other components and noise, and improve the reliability of gear's early fault diagnosis, a method of multiple information fusion based on single component feature (MIFSCF) is proposed. Vibration signal is sampled by many sensors installed on different positions of transmission. Firstly, meshing frequency component is separated by Fractional Fourier Transform (FRFT) as single component signal, whose order envelop demodulation spectrum (OEDS) is calculated next, and the value of OEDS at feature order of gear's fault is selected as feature parameter. Feature parameters of different sensors are fused by radar graph and the analysis results show that MIFSCF is more effective and accurate then the method based on single sensor and single parameter in gear fault diagnosis.

\section{Multiple information fusion based on single component feature}

\subsection{Definition of the FRFT}

For a signal $x(t)$, the FRFT can be defined as follow: 
$X_{p}(u)=F_{p}[x](u)=\int_{-\infty}^{\infty} x(t) K_{p}(t, u) d t$

The nucleus function of $X_{p}(u)$ is:

$K_{p}(t, u)=\left\{\begin{array}{l}A_{\alpha} \exp \left(j \pi \frac{t^{2}+u^{2}}{2} \cot \alpha-j \pi t u \csc \alpha\right), \quad \alpha \neq n \pi, \\ \delta(t-u), \quad \alpha=2 n \pi, \\ \delta(t+u), \quad \alpha=(2 n \pm 1) \pi,\end{array}\right.$

where $A_{\alpha}=\sqrt{(1-j \cot \alpha) / 2 \pi}, p$ is FRFT's important parameter, which can reflect the rotation angle in time-frequency distribution, and the rotating angle can be calculated by $\alpha=p \pi / 2$.

\subsection{Extraction of LFM signal based on FRFT}

A signal having many LFM components is difficult to be separated from each other either in time domain or frequency domain, as shown in Fig. 1. FRFT is able to rotate time-frequency distribution of signal by any angle, so if $\alpha$ and $\beta$ is orthogonal, the component $f=f_{0}+f_{m} t$ will be filtered from other components, and now FRFT's best order is $p_{0}=2 \alpha / \pi$.

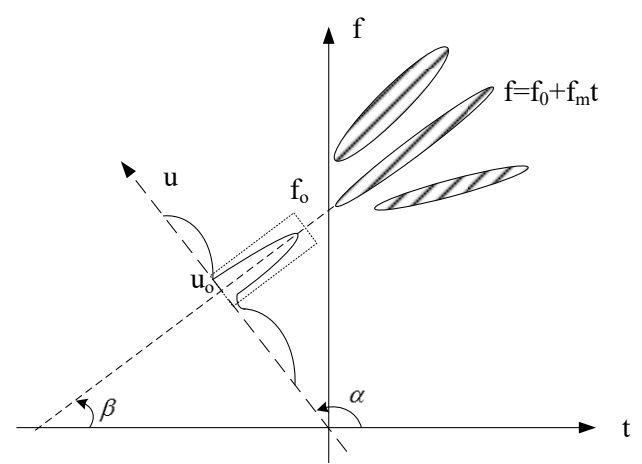

Fig. 1. Extraction of LFM signal based on FRFT

\subsection{Multiple information fusion based on single component feature}

Diagnosis of gear's early fault by analyzing only one sensor's signal is not accurate enough. So, a method of multiple information fusion based on single component feature (MIFSCF) is proposed. Vibration signal is sampled by many sensors installed on different positions of transmission. Meshing frequency component is separated by FRFT as single component signal $F_{i}$, whose OEDS is calculated next, and the value of OEDS at feature order of gear's fault is selected as feature parameter $P_{i} . P_{i}$ is fused by radar graph and MIFSCF is carried out.

\section{Platform of experiment and fault setting for gear}

Platform of experiment is based on a gearbox and vibration sensors are set at 8 different positions on gearbox, as shown in Fig. 2. An early failure of gear is simulated on second speed gear on output shaft, as shown in Fig. 3. The sample frequency is $20 \mathrm{kHz}$, and number of sampled data is 24576. Meshing order of second speed and constant speed (fourth speed) is 12.03 and 19, the fault feature order of gear on output shaft is 0.43 . 


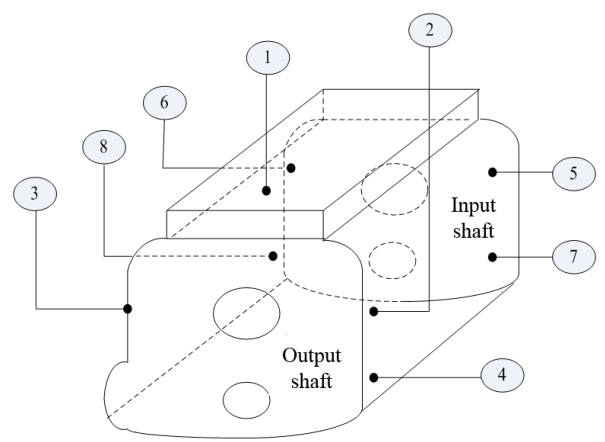

Fig. 2. Positions of vibration sensors

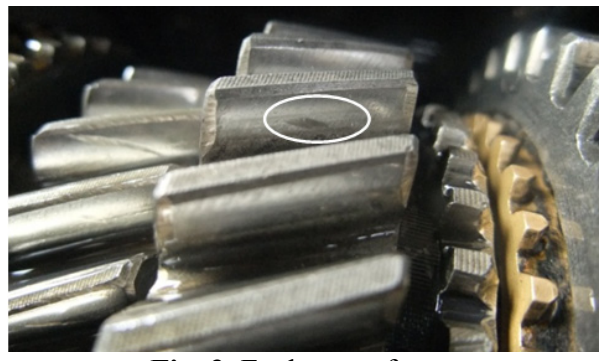

Fig. 3. Fault part of gear

\section{Diagnosis of gear's early fault based on MIFSCF}

\subsection{Separation of order components}

Rotation frequency and meshing frequency curves of different speeds are shown as Fig. 4. The time-frequency distribution of original vibration signal is shown as Fig. 5, it is difficult to distinguish each component, because different components are mixed together.

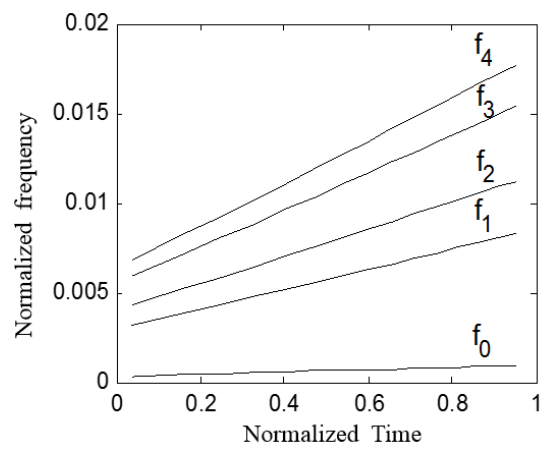

Fig. 4. Frequency curve of rotate speed and meshing component

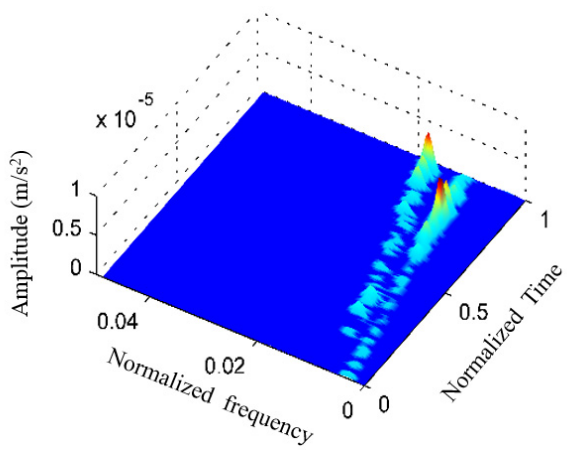

Fig. 6. Gabor time-frequency spectrum of separated components

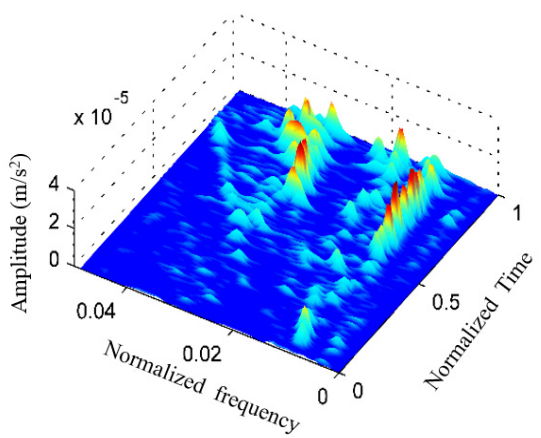

Fig. 5. Gabor time-frequency spectrum of vibration signal

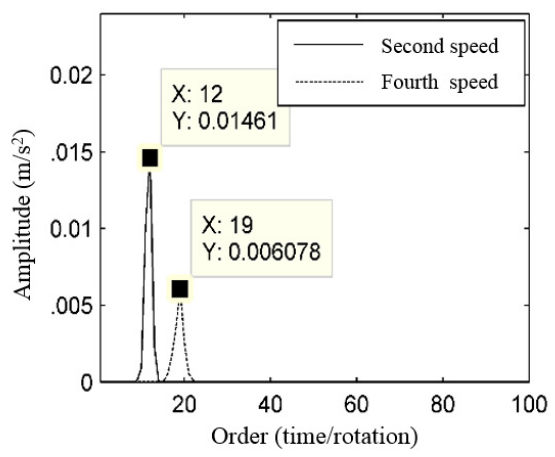

Fig. 7. Order spectrum of separated components

Second speed and fourth speed component are filtered by FRFT separately, the result is added together and Gabor time-frequency distribution is shown as Fig. 6, in which second speed and fourth speed component is clearly separated from other components and noise. In order to test the validity of this method, order spectrum of filtered second speed and fourth speed component is 
calculated and shown as Fig. 7. Order 12 and 19 is corresponding to theoretical value of second speed and fourth speed, which proves that components separation based on FRFT is valid and correct.

\subsection{Extraction of fault feature}

OEDS of original vibration signal is calculated as shown in Fig. 8, in which there is no obvious energy amplitude at feature order 0.43 , so it is difficult for OEDS to extract the submerged feature of early fault. OEDS of second speed meshing component filtered by FRFT is shown as Fig. 9. Energy of order 0.4375 is evident and close to theoretical order 0.43 , which proves that the weak fault feature is effectively extracted by FRFT.

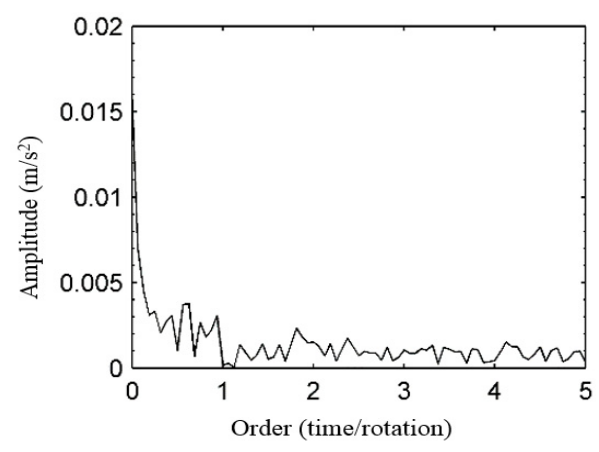

Fig. 8. OEDS of original vibration signal

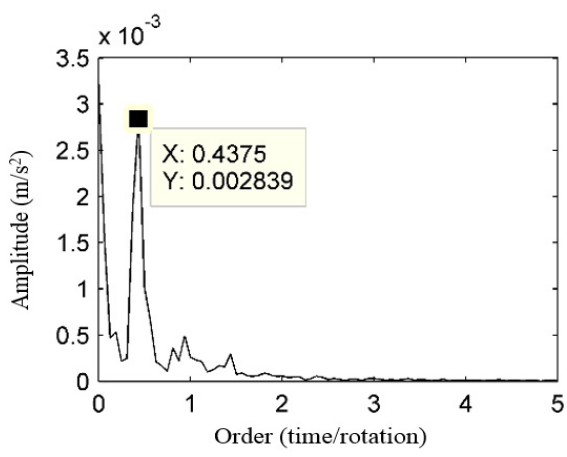

Fig. 9. OEDS of second speed signal extracted by FRFT

\subsection{Diagnosis based on MIFSCF}

8 sensors' signal of gear in normal state and fault state is separately filtered by FRFT, then OEDS of filtered components are calculated, and feature parameters are listed in Table 1.

Table 1. Feature parameters of normal and fault states $\left(\mathrm{m} / \mathrm{s}^{2}\right)$

\begin{tabular}{|c|c|c|c|c|c|c|c|c|}
\hline Sample & P1 & P2 & P3 & P4 & P5 & P6 & P7 & P8 \\
\hline Normal & 1.75 & 1.98 & 1.92 & 1.48 & 1.42 & 1.48 & 1.52 & 1.58 \\
\hline Fault & 2.83 & 2.78 & 2.68 & 1.78 & 1.63 & 1.68 & 1.47 & 1.44 \\
\hline
\end{tabular}

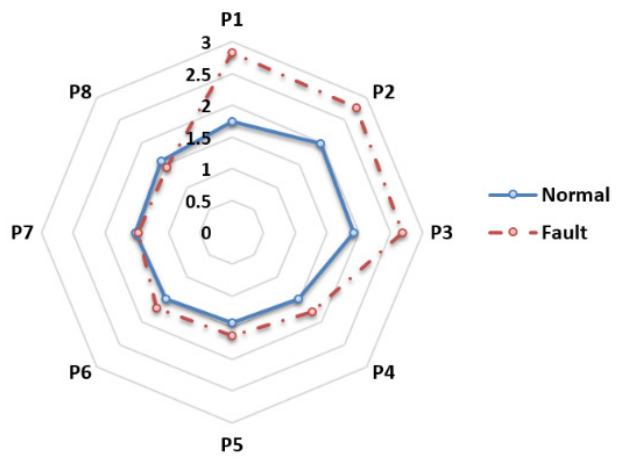

Fig. 10. Diagnosis based on MIFSCF

Parameters in Table 1 can't show the diagnosis result of gear early fault directly. So, parameters in Table 1 are fused by radar graph and shown as Fig. 10. Fig. 10 shows that P1, P2, P3 of fault are clearly different from normal state, so normal and fault state are clearly separated. P4, P5, P6 of fault are so close to normal state that difficult to be extinguished from each other. 
P7 and P8 of normal and fault state are crossed and can't be used to determine a fault is occurred. So MIFSCF is more effective in diagnosis by fusing multiple information of different sensors rather than only one sensor's information.

\section{Conclusions}

1) Components can be effectively separated by FRFT and other components and noise are removed simultaneously.

2) MIFSCF is effective in diagnosis of gear's early fault by fusing multiple information of different sensors.

\section{References}

[1] Ding Y., Sun L., Zhang H., et al. A multi-component LFM signal parameters estimation method using STFT and Zoom-FRFT. International Conference on Communication Software and Networks, 2016, p. 112-117.

[2] Song P., He Y., Cui W. Statistical property feature extraction based on FRFT for fault diagnosis of analog circuits. Analog Integrated Circuits and Signal Processing, Vol. 87, Issue 3, 2016, p. 427-436.

[3] Guo X., Shen Y., Yang S. Application of sample entropy and Fractional Fourier transform in the fault diagnosis of rolling bearings. Journal of Vibration and Shock, Vol. 36, Issue 18, 2017, p. 65-69.

[4] Lin L. F., Yu L., Wang H., et al. Parameter-adjusted stochastic resonance system for the aperiodic echo chirp signal in optimal FrFT domain. Communications in Nonlinear Science and Numerical Simulation, Vol. 43, 2017, p. 171-181.

[5] Zhai W., Zhang Y. Velocity measurement and radar imaging of high-speed target by using steppedchirp signal. Journal of Applied Remote Sensing, Vol. 8, Issue 7, 2014, p. 83-90.

[6] Mei J., Jia J., Zeng R., et al. FRFT filtering based on frequency tracking and extraction of gear's weak fault features. Journal of Vibration and Shock, Vol. 35, Issue 23, 2016, p. 130-135.

[7] Mei J. M., Chen X. L., Xiao Y. K., et al. Weak fault characteristic extraction based on steady holographic-order spectrum. Journal of Vibration and Shock, Vol. 31, Issue 12, 2012, p. 49-52.

[8] Liu R., Bao-Jie X. U. A study of the equipment fault diagnosing method based on EMD and holo-spectrum. International Journal of Plant Engineering and Management, Vol. 16, Issue 1, 2011, p. 28-34. 